\title{
A Pontryagin Maximum Principle for systems of flows
}

\author{
Héctor J. Sussmann \\ Department of Mathematics \\ Rutgers University \\ U.S.A. \\ sussmann@math . rutgers . edu
}

We present a generalization of the Pontryagin Maximum Principle, in which the usual adjoint equation, which contains derivatives of the system vector fields with respect to the state, is replaced by an integrated form, containing only differentials of the reference flow maps. In this form, the conditions of the maximum principle make sense for a number of control dynamical laws whose right-hand side can be nonsmooth, nonlipschitz, and even discontinuous. The "adjoint vectors" that are solutions of the "adjoint equation" no longer need to be absolutely continuous, and may be discontinuous and unbounded. We illustrate this with two examples: the "reflected brachistochrone problem" (RBP), and the derivation of Snell's law of refraction from Fermat's minimum time principle. In the RBP, where the dynamical law is Hölder continuous with exponent $1 / 2$, the adjoint vector turns out to have a singularity, in which one of the components goes to infinity from both sides, at an interior point of the interval of definition of the reference trajectory. In the refraction problem, where the dynamical law is discontinuous, the adjoint vector is bounded but has a jump discontinuity.

\section{Introduction}

It is well known that the minimum time problem whose solution is Snell's law of refraction was the first link of a long chain of mathematical developments that eventually led to the Pontryagin Maximum Principle (PMP) of optimal control theory: Snell's law was used by Johann Bernoulli's in his solution of the brachistochrone problem; this in turn was a decisive step towards the formulation of the general necessary condition of Euler and Lagrange for the classical Calculus of Variations; the Euler-Lagrange conditions were then strengthened by Legendre, whose second-order condition was later strengthened by Weierstrass; and, finally, Weierstrass' excess function condition led to the Pontryagin Maximum Principle (PMP), stated and proved in [1]. 
In the five decades since the formulation of the PMP, the result has been generalized in many directions, incorporating high-order conditions(cf. [7], [8]) and various types of nonsmoothness (cf. [2], [3], [4], [5], [6], [9],[13]), and producing intrinsic coordinate-free formulations on manifolds. It is remarkable and somewhat disappointing, however, that the refraction problem that leads to Snell's law does not fit within the framework of any of these generalizations, because even the non-smooth versions of the PMP require Lipschitz conditions on the system vector fields, and for the refraction problem the vector fields are actually discontinuous. A similar phenomenon occurs with the "reflected brachistochrone problem" (RBP), a very natural optimization problem with a Hölder continuous right-hand side.

The purpose of this note is to present a generalization of the PMP that applies to problems such as refraction ${ }^{1}$ and the RBP. This result - of which a preliminary announcement was made in 2004 in [12] — is a special case of several far-reaching extensions of the PMP proved by us in other papers (cf. $[10,11,12])$ that are much longer and more technical. We choose to isolate this particular aspect of the general results and present it separately because it lends itself to a relatively simple and self-contained treatment.

In our version of the PMP, the usual adjoint equation, which contains derivatives with respect to the state, is replaced by an integrated form, containing only differentials of the reference flow maps. In this form, the conditions of the maximum principle make sense for a number of control dynamical laws whose right-hand side can be nonsmooth, nonlipschitz, and even discontinuous. The "adjoint vectors" that are solutions of the "adjoint equation" no longer need to be absolutely continuous, and could even be discontinuous and unbounded. In both the refraction problem and the RBP, the state space is $\mathbb{R}^{2}$, and the system vector fields are smooth everywhere, except along the $x$ axis. For the refraction problem, the system vector fields are discontinuous, and the adjoint vector turns out to be discontinuous as well, but bounded, having a jump discontinuity at the point where the trajectory crosses the $x$ axis. axis. For the RBP, the system vector fields are Hölder continuous with exponent $1 / 2$, and - somehwat surprisingly, considering that the RBP vector fields are less irregular than those of the refraction problemthe adjoint vector turns out to be discontinuous with a worse singularity: at the point where the trajectory crosses the $x$ axis, the adjoint vector becomes infinite. And for both problems, the adjoint vector cannot possibly be characterized as a solution of an ordinary differential equation.

\footnotetext{
${ }^{1}$ Some readers may object to our inclusion of the refraction example here, on the grounds that the solution can easily be found by elementary means. Our motivation is identical to that of many authors of calculus of variations textbooks, who choose to include, as an application of the Euler-Lagrange equations, the derivation of the fact that the shortest path joining two points is a straight line segment, even though this can also be proved by completely trivial arguments. In both cases, the purpose is to show that the new necessary condition applies to a very old problem that played a key role in the early history of the subject.
} 


\section{Preliminaries on sets, maps, and flows}

Sets and maps. If $S$ is a set, then $\mathbb{I}_{S}$ will denote the identity map of $S$. If $A, B$ are sets, then the notations $f: A \hookrightarrow B, f: A \mapsto B$ will indicate, respectively, that $f$ is a possibly partially defined (abbr. "ppd") map from $A$ to $B$ and that $f$ is an everywhere defined map from $A$ to $B$. If $A \subseteq B$ and $f: B \hookrightarrow C$, then $f\lceil A$ is the restriction of $f$ to $A$, so $f\lceil A: A \hookrightarrow C$, and $f\lceil A: A \mapsto C$ if $f: B \mapsto C$.

Totally ordered sets. If $E$ is a totally ordered set, with ordering $\preceq$, we use $E^{\preceq, 2}$ to denote the set of all ordered pairs $(s, t) \in E \times E$ such that $s \preceq t$, and write $E^{\preceq, 3}$ to denote the set of all ordered triples $(r, s, t) \in E \times E \times E$ such that $r \preceq s \preceq t$. A subinterval of $E$ is a subset $I$ of $E$ such that, whenever $x \preceq y \preceq z, x \in I, z \in I$, and $y \in E$, it follows that $y \in I$. If $a \in E, b \in E$, and $a \preceq b$, then the $E$-interval from $a$ to $b$ is the set $[a, b]_{E} \stackrel{\text { def }}{=}\{x \in E: a \preceq x \preceq b\}$.

Manifolds, tangent and cotangent spaces. "Manifold" will mean "smooth manifold", "smooth" means "of class $C^{\infty}$," and $T_{x} M, T_{x}^{*} M$ denote, respectively, the tangent and cotangent spaces to a manifold $M$ at a point $x$ of $M$.

Set separation. Let $S_{1}$ and $S_{2}$ be subsets of a Hausdorff topological space $T$, and let $p$ be a point of $T$. We say that $S_{1}$ and $S_{2}$ are separated at $p$ if $S_{1} \cap S_{2} \subseteq\{p\}$, i.e. if $S_{1}$ and $S_{2}$ have no common point other than $p$. We say that $S_{1}$ and $S_{2}$ are locally separated at $p$ if there exists a neighborhood $V$ of $p$ such that $S_{1} \cap V$ and $S_{2} \cap V$ are separated.

Flows and their trajectories. Every sufficiently well-behaved vector field gives rise to a flow, but flows are typically less well-behaved than the vector fields that generate them. This is a reason for studying flows independently from their generators, as we now do.

Definition 1. Let $E$ be a totally ordered set with ordering $\preceq$, and let $\Omega$ be a set. A flow on $\Omega$ with time set $E$ (or, more simply, a flow on $(\Omega, E)$ ) is a family $\Phi=\left\{\Phi_{t, s}\right\}_{(s, t) \in E \preceq, 2}$ of ppd maps from $\Omega$ to $\Omega$ that satisfy the identities (F1) $\Phi_{t, s} \circ \Phi_{s, r}=\Phi_{t, r}$ whenever $(r, s, t) \in E^{\preceq, 3}$, and (F2) $\Phi_{t, t}=\mathbb{I}_{\Omega}$ whenever $t \in E$.

A trajectory of a flow $\Phi$ on $(\Omega, E)$ is a map $\xi: I \mapsto \Omega$, defined on a subinterval $I$ of $E$, such that $\xi(t)=\Phi_{t, s}(\xi(s))$ whenever $(s, t) \in I^{\preceq, 2}$.

Real augmentation of sets. If $\Omega$ is a set, then we will write $\Omega^{\#}=\mathbb{R} \times \Omega$. If $\Omega$ is a smooth manifold, then $\Omega^{\#}$ is obviously a smooth manifold as well. In that case, if $x^{\#}=\left(x_{0}, x\right) \in \Omega^{\#}$, the tangent space $T_{x \#} \Omega^{\#}$ and the cotangent space $T_{x}^{*} \Omega^{\#}$ will be identified with the products $\mathbb{R} \times T_{x} \Omega$ and $\mathbb{R} \times T_{x}^{*} \Omega$ using the canonical identification maps.

Augmented flows and their trajectories. In optimal control theory, it is often customary to "add the cost variable to the state of a system," thus transforming the optimization problem into a set separation problem in one higher dimension. This augmentation procedure can be carried out directly for flows. 
Definition 2. If $\Phi$ is a flow on $(\Omega, E)$, a real augmentation of $\Phi$ is a family $c=\left\{c_{t, s}\right\}_{(s, t) \in E \preceq, 2}$ of ppd functions from $\Omega$ to $\mathbb{R}$ such that

(RA) $c_{t, r}(x)=c_{s, r}(x)+c_{t, s}\left(\Phi_{s, r}(x)\right)$ whenever $x \in \Omega$ and $(r, s, t) \in E^{\preceq, 3}$.

A flow-augmentation pair (abbr. F-A pair) on $(\Omega, E)$ is a pair $(\Phi, c)$ such that $\Phi$ is a flow on $\Omega$ with time set $E$ and $c$ is a real augmentation of $\Phi$. $\diamond$

(Notice that (RA) implies, in particular, that $c_{t, t}(x)=0$, since we can always take $r=s=t$ and use the fact that $\Phi_{t, t}(x)=x$.)

To any F-A pair $(\Phi, c)$ on $(\Omega, E)$ we can associate a family of mappings $\Phi_{t, s}^{\#, c}: \Omega^{\#} \hookrightarrow \Omega^{\#}$, by letting $\Phi_{t, s}^{\#, c}\left(x_{0}, x\right)=\left(x_{0}+c_{t, s}(x), \Phi_{t, s}(x)\right)$ for each $(s, t) \in E^{\preceq, 2}$. It is then clear that $\Phi^{\#, c}=\left\{\Phi_{t, s}^{\#, c}\right\}_{(s, t) \in E \preceq, 2}$ is a flow on $\Omega^{\#}$. A flow $\Psi$ such that $\Psi=\Phi^{\#, c}$ for some $\Phi, c$ is called a real-augmented flow. It is easy to see that a flow $\Psi=\left\{\Psi_{t, s}\right\}_{(s, t) \in E \preceq, 2}$ on $\mathbb{R} \times \Omega$ is a real-augmented flow if and only if -if we write $\Psi_{t, s}\left(x_{0}, x\right)=\left(\psi_{0, t, s}\left(x_{0}, x\right), \psi_{t, s}\left(x_{0}, x\right)\right)$ - the maps $\psi_{0, t, s}, \psi_{t, s}$ are such that the point $\psi_{t, s}\left(x_{0}, x\right) \in \Omega$ and the number $\psi_{0, t, s}\left(x_{0}, x\right)-x_{0} \in \mathbb{R}$ do not depend on $x_{0}$. In that case, the pair $(\Phi, c)$ such that $\Psi=\Phi^{\#, c}$ is uniquely determined by $\Psi$ as follows: $\Phi_{t, s}(x)=\psi_{t, s}\left(x_{0}, x\right)$ and $c_{t, s}(x)=\psi_{0, t, s}\left(x_{0}, x\right)-x_{0}$ if $\left(x_{0}, x\right) \in \Omega^{\#}$.

Definition 3. An augmented trajectory of an F-A pair $(\Phi, c)$ on a pair $(\Omega, E)$ is a trajectory of the flow $\Phi^{\#, c}$, i. e., a map $I \ni t \mapsto \xi^{\#}(t)=\left(\xi_{0}(t), \xi(t)\right) \in \Omega^{\#}$, defined on a subinterval $I$ of $E$, such that $\xi(t)=\Phi_{t, s}(\xi(s))$ and $\xi_{0}(t)=$ $\xi_{0}(s)+c_{t, s}(\xi(s))$ whenever $(s, t) \in I^{\preceq, 2}$.

Differentiability of flows. Given a trajectory $\xi$ of a flow $\Phi$, it makes sense to talk about differentiability of $\Phi$ along $\xi$.

Definition 4. Assume that $\Omega$ is a manifold, $\Phi=\left\{\Phi_{t, s}\right\}_{(s, t) \in E \preceq, 2}$ is a flow on $(\Omega, E), I$ is a subinterval of $E$, and $\xi: I \mapsto \Omega$ is a trajectory of $\Phi$. We call $\Phi$

(1) continuous near $\xi$ if for each $(s, t) \in I^{\preceq, 2}$ the map $\Phi_{t, s}$ is continuous on a neighborhood of $\xi(s)$.

(2) differentiable along $\xi$ if for each $(s, t) \in I^{\preceq, 2}$ the flow map $\Phi_{t, s}$ is differentiable at $\xi(s)$.

The above definition can be applied to an augmented flow $\Phi^{\#, c}$. If $\Phi^{\#, c}$ is differentiable along an augmented trajectory $\xi^{\#}=\left(\xi_{0}, \xi\right)$, then the differentials $D \Phi_{t, s}^{\#, c}\left(\xi^{\#}(s)\right)$ have a special structure, reflecting the special structure of the maps $\Phi_{t, s}^{\#, c}$. Indeed, if we write $M_{t, s}^{\#}=D \Phi_{t, s}^{\#, c}\left(\xi^{\#}(s)\right)$ (so that $M_{t, s}^{\#}$ is a linear map from $T_{\xi^{\#}(s)} \Omega^{\#}$ to $\left.T_{\xi^{\#(t)}} \Omega^{\#}\right)$, then it is easy to see that the result $M_{t, s \mid}^{\#} \cdot\left(v_{0}, v\right)$ of applying the linear map $D \Phi_{t, s}^{\#, c}\left(\xi^{\#}(s)\right)$ to a tangent vector $\left(v_{0}, v\right) \in T_{\xi \#(s)} \Omega^{\#} \sim \mathbb{R} \times T_{\xi(s)} \Omega$ is the vector $\left(v_{0}+m_{t, s} \cdot v, M_{t, s} \cdot v\right)$, which belongs to $T_{\xi^{\#}(t)} \Omega^{\#} \sim \mathbb{R} \times T_{\xi(t)} \Omega$, where

$$
m_{t, s}=D c_{t, s}(\xi(s)), \quad M_{t, s}=D \Phi_{t, s}(\xi(s)),
$$

so that $m_{t, s} \in T_{\xi(s)}^{*} \Omega$ and $M_{t, s}$ is a linear map from $T_{\xi(s)} \Omega$ to $T_{\xi(t)} \Omega$. 
Variational fields. The differentials $D \Phi_{t, s}(\xi(s)), D \Phi_{t, s}^{\#}\left(\xi^{\#}(s)\right)$, can be used to propagate tangent vectors forwards and cotangent vectors backwards.

Definition 5. A field of tangent vectors $I \ni s \mapsto v(s) \in T_{\xi(s)} \Omega$ such that $v(t)=M_{t, s} \cdot v(s)$ whenever $(s, t) \in I^{\preceq, 2}$ —where $M_{t, s}$ is defined by (1) -is called a variational vector field of $(\Phi, c)$ along $\xi$.

Definition 6. A field of vectors $I \ni s \mapsto v^{\#}(s)=\left(v_{0}(s), v(s)\right) \in T_{\xi^{\#}(s)} \Omega^{\#}$ such that $v_{0}(t)=v_{0}(s)+m_{t, s} \cdot v(s)$ and $v(t)=M_{t, s} \cdot v(s)$ whenever $(s, t) \in I^{\preceq, 2}$-where $m_{t, s}, M_{t, s}$ are defined by (1) - is called an augmented variational vector field of $(\Phi, c)$ along $\xi$.

Adjoint fields. The dual maps $\Lambda_{t, s}^{\#} \stackrel{\text { def }}{=}\left(D \Phi_{t, s}^{\#, c}(\xi(s))\right)^{\dagger}: T_{\xi^{\#}(t)}^{*} \Omega^{\#} \mapsto T_{\xi^{\#}(s)}^{*} \Omega^{\#}$ (where, naturally, we use the canonical identification $T_{\xi^{\#}(r)}^{*} \Omega^{\#} \sim \mathbb{R} \times T_{\xi(r)}^{*} \Omega$ for every $r$ ) are given (if we write the maps as acting on the right on augmented covectors) by $\left.\left(\omega^{\#}(t) \cdot \Lambda_{t, s}^{\#}\right) \cdot v^{\#}(s)=\omega^{\#}(t) \cdot M_{t, s}^{\#} v^{\#}(s)\right)$, so that, if we write $\omega^{\#}(t)=\left(\omega_{0}(t), \omega(t)\right)$, we see that

$$
\begin{aligned}
\left(\omega_{0}(s), \omega(s)\right) \cdot\left(v_{0}(s), v(s)\right) & =\left(\left(\omega_{0}(t), \omega(t)\right) \cdot \Lambda_{t, s}^{\#}\right) \cdot\left(v_{0}(s), v(s)\right) \\
& =\omega_{0}(t) v_{0}(s)+\omega_{0}(t) m_{t, s} \cdot v(s)+\left(\omega(t) \circ M_{t, s}\right) \cdot v(s),
\end{aligned}
$$

and then $\omega_{0}(s)=\omega_{0}(t)$ and $\omega(s)=\omega_{0} m_{t, s}+\omega(t) \circ M_{t, s}$.

Definition 7. A field of covectors $I \ni s \mapsto \omega^{\#}(s)=\left(\omega_{0}(s), \omega(s)\right) \in T_{\xi^{\#}(s)}^{*} \Omega^{\#}$ such that $\omega_{0}$ is a constant function, and $\omega$ satisfies

$$
\omega(s)=\omega_{0} m_{t, s}+\omega(t) \circ M_{t, s} \text { whenever }(s, t) \in I^{\preceq, 2},
$$

is called an augmented adjoint field of covectors (or augmented adjoint vector) of $(\Phi, c)$ along $\xi$.

The constant $-\omega_{0}$ is the abnormal multiplier, and the identity (2) is the integrated adjoint equation.

Approximating cones. A cone in a real linear space $X$ is a nonempty subset of $X$ which is closed under multiplication by nonnegative scalars, i.e., such that if $c \in C, r \in \mathbb{R}$, and $r \geq 0$, it follows that $r c \in C$. (It then follows automatically that $0 \in C$.) The polar of a cone $C$ in $X$ is the set $C^{\dagger}=\left\{\lambda \in X^{\dagger}: \lambda(c) \leq 0\right.$ whenever $\left.c \in C\right\}$, where $X^{\dagger}$ is the dual space ${ }^{2}$ of $X$.

Definition 8. If $M$ is a smooth manifold, $S \subseteq M$, and $s \in S$, a Boltyanskii approximating cone to $S$ at $s$ is a convex cone $C$ in $T_{s} M$ having the property that there exist $m, U, D, F, L$, such that

\footnotetext{
${ }^{2}$ In all cases occurring in this paper, $X$ is finite-dimensional, so we do not need to distinguish between algebraic and topological duals.
} 
(1) $m \in \mathbb{N}, U$ is a neighborhood of 0 in $\mathbb{R}^{m}$, and $D$ is a convex cone in $\mathbb{R}^{m}$

(2) $F: U \cap D \mapsto M$ is a continuous map such that $F(U \cap D) \subseteq S$,

(3) $L: \mathbb{R}^{m} \mapsto T_{s} M$ is a linear map,

(4) $F(v)-s-L \cdot v=o(\|v\|)$ as $v \rightarrow 0$ via values in $U \cap D$,

(5) $L \cdot D=C$.

\section{The main theorem}

We consider optimal control problems arising from an augmented flow system $\Psi=\left\{{ }_{\eta} \Psi\right\}_{\eta \in \mathcal{U}}$, indexed ${ }^{3}$ by a "class of admissible controls" $\mathcal{U}$. We assume that a fixed totally ordered set $E$ is specified, such that the time set $E_{\eta}$ of each ${ }_{\eta} \Psi$ is equal to $E$.

The state space of the system is a smooth manifold $\Omega$. Each ${ }_{\eta} \Psi$ is a flow on the real-augmented space $\Omega^{\#}=\mathbb{R} \times \Omega$, given by ${ }_{\eta} \Psi={ }_{\eta} \Phi^{\#},{ }_{\eta} c$, where the pair $\left({ }_{\eta} \Phi,{ }_{\eta} c\right)$ is a real-augmented flow on $\Omega$ with time set $E$. We use $\preceq$ to denote the ordering of $E$. We assume we are given an initial state $\hat{x} \in \Omega$, a terminal set $S$, which is a subset of $\Omega$, and initial and terminal times $\hat{a} \in E$, $\hat{b} \in E$, such that $\hat{a} \preceq \hat{b}$.

The objective is to minimize the $\operatorname{cost}{ }_{\eta} c_{\hat{b}, \hat{a}}(\hat{x})$ in the class $\mathcal{A}$ of all $\eta \in \mathcal{U}$ such that the terminal point ${ }_{\eta} \Phi_{\hat{b}, \hat{a}}(\hat{x})$ belongs to $S$. Equivalently, we want to minimize the cost $\xi_{0}(\hat{b})-\xi_{0}(\hat{a})$ in the class $\tilde{\mathcal{A}}$ of all pairs $\left(\eta, \xi^{\#}\right)$ such that $\eta \in \mathcal{U}, \xi^{\#}=\left(\xi_{0}, \xi\right)$ is an augmented trajectory of $\left({ }_{\eta} \Phi,{ }_{\eta} c\right), \xi(\hat{a})=\hat{x}$ and $\xi(\hat{b}) \in S$.

We assume that we are given data

$$
\mathcal{D}=(n, \Omega, E, \preceq, \mathcal{U}, \Phi, c, \Psi, \hat{a}, \hat{b}, \hat{x}, S),
$$

so that $\Psi=\left\{{ }_{\eta} \Psi\right\}_{\eta \in \mathcal{U}}, \Phi=\left\{{ }_{\eta} \Phi\right\}_{\eta \in \mathcal{U}}, c=\left\{{ }_{\eta} c\right\}_{\eta \in \mathcal{U}}$, and ${ }_{\eta} \Psi={ }_{\eta} \Phi^{\#,{ }_{\eta} c}$ for every $\eta \in \mathcal{U}$. We define $\hat{I}=\{t \in E: \hat{a} \preceq t \preceq \hat{b}\}$.

Precisely, we will assume that $\mathcal{D}$ satisfies

(A1) $n \in \mathbb{N}$, and $\Omega$ is a smooth manifold of dimension $n$;

(A2) $E$ is a totally ordered set, with partial ordering $\preceq$;

(A3) $\mathcal{U}$ is a set;

(A4) $\Psi=\left\{{ }_{\eta} \Psi\right\}_{\eta \in \mathcal{U}}$ is an augmented flow system on $\Omega$ with time set $E$;

(A5) ${ }_{\eta} \Psi={ }_{\eta} \Phi^{\#,{ }_{\eta} c}$, where $\left({ }_{\eta} \Phi,{ }_{\eta} c\right)$ is a flow-augmentation pair on $\Omega$ with time set $E$;

(A6) $\hat{x} \in \Omega, \hat{a} \in E, \hat{b} \in E$, and $\hat{a} \preceq \hat{b}$;

(A7) $\hat{a} \in E, \hat{b} \in E$, and $\hat{a} \preceq \hat{b}$.

\footnotetext{
${ }^{3}$ We put the subscript $\eta$ on the left because we will want to write formulas such as ${ }_{\eta} \Psi={ }_{\eta} \Phi^{\#, \eta_{\eta} c},{ }_{\eta} \Psi=\left\{{ }_{\eta} \Psi_{t, s}\right\}_{(s, t) \in E \preceq, 2}$ and ${ }_{\eta} \Phi^{\#, \eta_{\eta} c}=\left\{{ }_{\eta} \Phi_{t, s}^{\#, \eta^{c}}\right\}_{s, t \in E_{\eta}}$.
} 
We let $\tilde{\mathcal{A}}$ be the class of all pairs $\left(\eta, \xi^{\#}\right)$ such that $\eta \in \mathcal{U}, \xi^{\#}=\left(\xi_{0}, \xi\right)$ is an augmented trajectory of $\left({ }_{\eta} \Phi,{ }_{\eta} c\right), \xi(\hat{a})=\hat{x}$, and $\xi(\hat{b}) \in S$.

We assume that we are given a candidate control $\eta_{*}$ and candidate augmented trajectory $\xi_{*}^{\#}=\left(\xi_{*, 0}, \xi_{*}\right)$, such that

(A8) $\left(\eta_{*},\left(\xi_{*, 0}, \xi_{*}\right)\right)=\left(\eta_{*}, \xi_{*}^{\#}\right) \in \tilde{\mathcal{A}}$.

Clearly, then, the three maps $\xi_{*}: \hat{I} \mapsto \Omega$ (the "reference trajectory"), $\xi_{0, *}: \hat{I} \mapsto \mathbb{R} \quad$ (the "reference running cost"), $\quad \xi_{*}^{\#}: \hat{I} \mapsto \Omega^{\#}$ (the "reference augmented trajectory"), satisfy, for all $t \in \hat{I}$,

$$
\xi_{*}(t)={ }_{\eta} \Phi_{t, \hat{a}}\left(\hat{x}_{*}\right), \quad \xi_{0, *}(t)={ }_{\eta} c_{t, \hat{a}}\left(\hat{x}_{*}\right), \quad \xi_{*}^{\#}(t)={ }_{\eta} \Phi_{t, a}^{\#, \eta c}\left(0, \hat{x}_{*}\right),
$$

as well as $\xi_{*}^{\#}(t)=\left(\xi_{0, *}(t), \xi_{*}(t)\right)$.

Our key assumption is that the pair $\left(\eta_{*}, \xi_{*}^{\#}\right)$ is a solution of our optimal control problem, that is, that

(A9) $\xi_{*, 0}(\hat{b})-\xi_{*, 0}(\hat{a}) \leq \xi_{0}(\hat{b})-\xi_{0}(\hat{a})$ for all $\left(\eta,\left(\xi_{0}, \xi\right)\right) \in \tilde{\mathcal{A}}$.

In addition, we make the crucial technical assumption that

(A10) The reference flow $\eta_{*} \Psi$ is continuous near the reference trajectory $\xi_{*}^{\#}$, and differentiable along $\xi_{*}^{\#}$.

We define an impulse vector for the data 12 -tuple $\mathcal{D}$ and the reference control-augmented trajectory pair $\left(\eta_{*}, \xi_{*}^{\#}\right)$ to be a pair $\left(v^{\#}, t\right)$ such that $t \in \hat{I}$ and $v^{\#} \in T_{\xi_{*}^{*}(t)} \Omega^{\#}$. We use $\mathcal{V}_{\max }\left(\mathcal{D},\left(\eta_{*}, \xi_{*}^{\#}\right)\right)$ to denote the set of all impulse vectors for $\mathcal{D},\left(\eta_{*}, \xi_{*}^{\#}\right)$.

Let $\mathbf{v}^{\#}=\left(\left(v_{1}^{\#}, t_{1}\right), \ldots,\left(v_{m}^{\#}, t_{m}\right)\right)$ be a finite sequence of members of $\mathcal{V}_{\max }\left(\mathcal{D},\left(\eta_{*}, \xi_{*}^{\#}\right)\right)$, and assume that (A10) holds. We then define linear maps

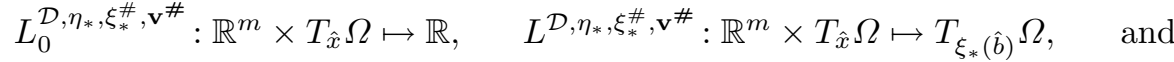
$L^{\#, \mathcal{D}, \eta_{*}, \xi_{*}^{\#}, \mathbf{v}^{\#}}: \mathbb{R}^{m} \times T_{\hat{x}} \Omega \mapsto T_{\xi_{*}(\hat{b})} \Omega^{\#}$, by first writing $v_{j}^{\#}=\left(v_{0, j}, v_{j}\right)$, with $v_{0, j} \in \mathbb{R}, v_{j} \in T_{\xi_{*}(t)} \Omega$, and then letting

$$
\begin{aligned}
& L_{0}^{\mathcal{D}, \eta_{*}, \xi_{*}^{\#}, \mathbf{v}^{\#}}\left(\varepsilon_{1}, \ldots, \varepsilon_{m}, w\right)=m_{\hat{b}, \hat{a}} \cdot w+\sum_{j=1}^{m} \varepsilon_{j}\left(v_{0, j}+m_{\hat{b}, t_{j}} \cdot v_{j}\right), \\
& L^{\mathcal{D}, \eta_{*}, \xi_{*}^{\#}, \mathbf{v}^{\#}}\left(\varepsilon_{1}, \ldots, \varepsilon_{m}, w\right)=M_{\hat{b}, \hat{a}} \cdot w+\sum_{j=1}^{m} \varepsilon_{j} M_{\hat{b}, t_{j}} \cdot v_{j}, \\
& L^{\#, \mathcal{D}, \eta_{*}, \xi_{*}^{\#}, \mathbf{v}^{\#}}\left(\varepsilon_{1}, \ldots, \varepsilon_{m}, w\right)= \\
&\left(L_{0}^{\mathcal{D}, \eta_{*}, \xi_{*}^{\#}, \mathbf{v}^{\#}}\left(\varepsilon_{1}, \ldots, \varepsilon_{m}, w\right), L^{\mathcal{D}, \eta_{*}, \xi_{*}^{\#}, \mathbf{v}}\left(\varepsilon_{1}, \ldots, \varepsilon_{m}, w\right)\right) .
\end{aligned}
$$

In the following definition, $\mathbb{R}_{+}^{m}$ denotes the nonnegative orthant of $\mathbb{R}^{m}$, that is, the set $\left\{\left(h_{1}, \ldots, h_{m}\right) \in \mathbb{R}^{m}: h_{1} \geq 0, \ldots, h_{m} \geq 0\right\}$. Furthermore, for 
any $a, b \in E$ such that $a \preceq b$, and any $x \in \Omega, \mathcal{R}_{a, b}^{\#}(x)$ denotes the "reachable set from $x$ for the augmented system over the interval from $a$ to $b$," so that

$$
\mathcal{R}_{a, b}^{\#}(x) \stackrel{\text { def }}{=}\left\{(r, y):(\exists \eta \in \mathcal{U})\left(y={ }_{\eta} \Phi_{b, a}(x), \text { and } r={ }_{\eta} c_{b, a}(x)\right)\right\} .
$$

Definition 9. A set $\mathcal{V}$ of impulse vectors is variational for $\mathcal{D}, \eta_{*}, \xi_{*}^{\#}$ if for every finite sequence $\mathbf{v}^{\#}=\left(\left(v_{1}^{\#}, t_{1}\right), \ldots,\left(v_{m}^{\#}, t_{m}\right)\right)$ of members of $\mathcal{V}$ it follows that there exist neighborhoods $P, Q$ of $0, \hat{x}$, in $\mathbb{R}_{+}^{m}, \Omega$, respectively, and a continuous map $F: P \times Q \mapsto \Omega^{\#}$, such that

(1) $F$ is differentiable at $(0, \hat{x})$ with differential $L^{\#, \mathcal{D}, \eta_{*}, \xi_{*}^{\#}, \mathbf{v}^{\#}}$ (in the precise sense of Remark 1 below).

(2) $F(P \times\{x\}) \subseteq \mathcal{R}_{\hat{a}, \hat{b}}^{\#}(x)$ for every $x \in Q$.

Remark 1. The precise meaning of the assertion that " $F$ is differentiable at

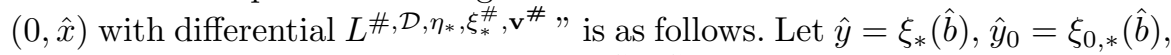
$\hat{y}^{\#}=\left(\hat{y}_{0}, \hat{y}\right)$, so $\hat{y}^{\#}=F(0, \hat{x})$. Let $\tilde{P}, \tilde{Q}, R, J$ be open neighborhoods of $0, \hat{x}, \hat{y}_{0}, \hat{y}$, in $\mathbb{R}^{m}, \Omega, \mathbb{R}, \Omega$, respectively, such that $\tilde{P} \subseteq P, \tilde{Q} \subseteq Q$, $F\left(\left(\tilde{P} \cap \mathbb{R}_{+}^{m}\right) \times \tilde{Q}\right) \subseteq J \times R, \tilde{Q}$ is the domain of a coordinate chart $\kappa: \tilde{Q} \mapsto \mathbb{R}^{n}$ for which $\kappa(\hat{x})=0$, and $R$ is the domain of a coordinate chart $\zeta: R \mapsto \mathbb{R}^{n}$ for which $\zeta(\hat{y})=0$. Use $\kappa$ and $\zeta$ to identify the sets $\tilde{Q}$ and $R$ with their images $\kappa(\tilde{Q}), \zeta(R)$, so $\tilde{Q}$ and $R$ are now open subsets of $\mathbb{R}^{n}$. Then

$$
F\left(\varepsilon_{1}, \ldots, \varepsilon_{m}, w\right)=L^{\#, \mathcal{D}, \eta_{*}, \xi_{*}^{\#}, \mathbf{v}^{\#}}\left(\varepsilon_{1}, \ldots, \varepsilon_{m}, w\right)+o\left(\varepsilon_{1}+\ldots+\varepsilon_{m}+\|w\|\right)
$$

as $\left(\varepsilon_{1}, \ldots, \varepsilon_{m}, w\right)$ goes to 0 via values in $\left(\tilde{P} \cap \mathbb{R}_{+}^{m}\right) \times \tilde{Q}$.

Our last two assumptions are

$(\mathrm{A} 11) \mathcal{V}$ is a variational set of impulse vectors for $\mathcal{D}, \eta_{*}, \xi_{*}^{\#}$.

(A12) $C$ is a Boltyanskii approximating cone to $S$ at $\xi_{*}(\hat{b})$.

The following is then our main result.

Theorem 1. Assume that we are given a data 12-tuple $\mathcal{D}$ as in (3), as well as $\eta_{*}, \xi_{*}^{\#}, \mathcal{V}, C$, such that Assumptions (A1) to (A12) hold. Write

$$
\Phi_{t, s}^{*}={ }_{\eta_{*}} \Phi_{t, s}, \quad c_{t, s}^{*}={ }_{\eta_{*}} c_{t, s}, \quad M_{t, s}=D \Phi_{t, s}^{*}\left(\xi_{*}(s)\right), \quad m_{t, s}=D c_{t, s}^{*}\left(\xi_{*}(s)\right),
$$

(so that $m_{t, s}=\nabla c_{t, s}^{*}\left(\xi_{*}(s)\right)$ ). Then there exist a map $\hat{I} \ni t \mapsto \omega(t) \in \mathbb{R}_{n}$ and a real constant $\omega_{0}$ such that

(1) $\omega_{0} \geq 0$

(2) $\left(\omega_{0}, \omega(t)\right) \neq(0,0)$ for all $t \in I$,

(3) $\omega(s)=\omega(t) \cdot M_{t, s}-\omega_{0} m_{t, s}$ whenever $s, t \in E$ and $s \leq t$,

(4) $\langle\omega(t), v\rangle-\omega_{0} v_{0} \leq 0$ whenever $\left(v^{\#}, t\right)=\left(\left(v_{0}, v\right), t\right) \in \mathcal{V}$, 


$$
\text { the transversality condition }-\omega(\hat{b}) \in C^{\dagger} \text { holds. }
$$

Proof. Fix a norm $\|\cdot\|$ on the tangent space $T_{\xi_{*}(\hat{b})} \Omega$. Let $\mathcal{K}$ be the set of all pairs $\left(\tilde{\omega}_{0}, \bar{\omega}\right) \in \mathbb{R} \times T_{\xi_{*}(\hat{b})} \Omega$ such that $\tilde{\omega}_{0} \leq 0,\left|\tilde{\omega}_{0}\right|+\|\bar{\omega}\|=1$, and $-\bar{\omega} \in C^{\dagger}$. Then $\mathcal{K}$ is clearly compact. For each subset $\mathcal{W}$ of $\mathcal{V}$, let $\mathcal{K}(\mathcal{W})$ be the subset of $\mathcal{K}$ consisting of all $\left(\tilde{\omega}_{0}, \bar{\omega}\right) \in \mathcal{K}$ such that, for all $\left(\left(v_{0}, v\right), t\right) \in \mathcal{W}$, the identity $\left\langle\bar{\omega} \cdot D_{\eta_{*}} \Phi_{\hat{b}, t}\left(\xi_{*}(t)\right)+\tilde{\omega}_{0} \nabla c_{\hat{b}, t}\left(\xi_{*}(t)\right), v\right\rangle+\tilde{\omega}_{0} v_{0} \leq 0$ holds.

It clearly suffices to prove that the set $\mathcal{K}(\mathcal{V})$ is nonempty. Indeed, if a pair $\left(\tilde{\omega}_{0}, \bar{\omega}\right)$ belongs to $\in \mathcal{K}(\mathcal{V})$, we may define $\omega_{0}=-\tilde{\omega}_{0}$ and then, for for $t \in I$, let $\omega(t)=\bar{\omega} \cdot M_{\hat{b}, t}+\tilde{\omega}_{0} m_{\hat{b}, t}$ (that is, $\left.\omega(t)=\bar{\omega} \cdot M_{\hat{b}, t}-\omega_{0} m_{\hat{b}, t}\right)$ for $t \in I$. Write $\omega^{\#}=\left(\omega_{0}, \omega\right)$. A simple calculation shows that $\omega^{\#}$ is an augmented adjoint vector that satisfies all our conclusions.

Furthermore, it is evident from the definition of the sets $\mathcal{K}(\mathcal{W})$ that if a subset $\mathcal{W}$ of $\mathcal{V}$ is the union $\cup_{\lambda \in \Lambda} \mathcal{W}_{\lambda}$ of a family of subsets $\mathcal{V}$, then

$$
\mathcal{K}(\mathcal{W})=\cap_{\lambda \in \Lambda} \mathcal{K}\left(\mathcal{W}_{\lambda}\right)
$$

Hence it suffices to prove that $\mathcal{K}(\mathcal{W})$ is nonempty whenever $\mathcal{W}$ is a finite subset of $\mathcal{V}$.

So let $\mathcal{W}$ be a finite subset of $\mathcal{V}$. Let $\mathbf{v}^{\#}=\left(\left(v_{1}^{\#}, t_{1}\right), \ldots,\left(v_{m}^{\#}, t_{m}\right)\right)$ be a finite sequence that contains all the members of $\mathcal{W}$, and write $v_{j}^{\#}=\left(v_{0, j}, v_{j}\right)$ for $j=1, \ldots, m$. Since $\mathcal{V}$ is variational, Definition 3 enables us to pick neighborhoods $P, Q, R$ of $0, \hat{x}\left(=\xi_{*}(\hat{a})\right), \xi_{*}^{\#}(\hat{b})$, in $\mathbb{R}_{+}^{m}, \Omega, \Omega^{\#}$, respectively, and a continuous map $F: P \times Q \mapsto R$, which is differentiable at $(0, \hat{x})$ with

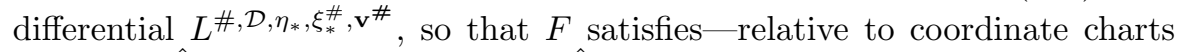
near $\hat{x}, \xi_{*}(\hat{b})$ for which $\hat{x}=0$ and $\xi_{*}(\hat{b})=0$ - the condition

$$
F\left(\varepsilon_{1}, \ldots, \varepsilon_{m}, w\right)=L^{\#, \mathcal{D}, \eta_{*}, \xi_{*}^{\#}, \mathbf{v}^{\#}}\left(\varepsilon_{1}, \ldots, \varepsilon_{m}, w\right)+o\left(\varepsilon_{1}, \ldots, \varepsilon_{m}+\|w\|\right)
$$

as $\left(\varepsilon_{1}, \ldots, \varepsilon_{m}, w\right)$ goes to $(0,0)$ via values in $\mathbb{R}_{+}^{m} \times Q$, as well as the property that $F(P \times\{x\}) \subseteq \mathcal{R}_{\hat{a}, \hat{b}}^{\#}(x)$ for every $x \in Q$.

In particular, if we let $G: P \mapsto \Omega^{\#}$ be the map given by

$$
G\left(\varepsilon_{1}, \ldots, \varepsilon_{m}\right)=F\left(\varepsilon_{1}, \ldots, \varepsilon_{m} ; 0\right),
$$

then $G$ is a continuous map into $\mathcal{R}_{\hat{a}, \hat{b}}^{\#}(\hat{x})$ which is differentiable at 0 with

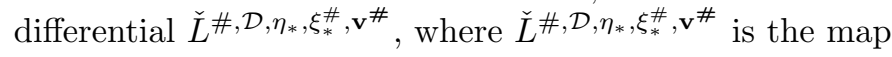

$$
\left(\varepsilon_{1}, \ldots, \varepsilon_{m}\right) \mapsto\left(\sum_{j=1}^{m} \varepsilon_{j}\left(v_{0, j}+m_{\hat{b}, t_{j}} \cdot v_{j}\right), \sum_{j=1}^{m} \varepsilon_{j} M_{\hat{b}, t_{j}} \cdot v_{j}\right),
$$

and $M_{t, s}, m_{t, s}$, are defined by

$$
M_{t, s}=D \Phi_{t, s}\left(\xi_{*}(s)\right), \quad m_{t, s}=\nabla c_{t, s}\left(\xi_{*}(s)\right) \text { for } s, t \in I, s \preceq t .
$$


Let $w_{j}^{\#}=\check{L}^{\#, \mathcal{D}, \eta_{*}, \xi_{*}^{\#}, \mathbf{v}^{\#}} \cdot e_{j}^{m}$, where $e_{j}^{m}=\left(\delta_{j}^{1}, \ldots, \delta_{j}^{m}\right)$, and the $\delta_{j}^{k}$ are the Kronecker symbols. Then $w_{j}^{\#}=\left(v_{0, j}+m_{\hat{b}, t_{j}} \cdot v_{j}, M_{\hat{b}, t_{j}} \cdot v_{j}\right)$ and and $\check{L}^{\#, \mathcal{D}, \eta_{*}, \xi_{*}^{\#}, \mathbf{v}^{\#}}\left(\varepsilon_{1}, \ldots, \varepsilon_{m}\right)=\sum_{j=1}^{m} \varepsilon_{j} w_{j}^{\#}$.

It is then clear (by applying Definition 8 , with $D=\mathbb{R}_{+}^{m}, L=\check{L}^{\#, \mathcal{D}, \eta_{*}, \xi_{*}^{\#}, \mathbf{v}^{\#}}$ ) that, if we write $\mathcal{C}$ to denote the convex cone $\mathcal{C}$ generated by the vectors $w_{j}^{\#}$

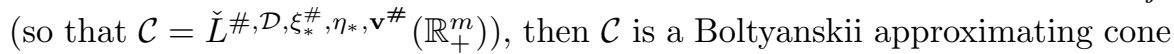
to the augmented reachable set $\mathcal{R}_{\hat{a}, \hat{b}}^{\#}(\hat{x})$ at $\xi_{*}^{\#}(\hat{b})$.

Now, let $S^{\#}=\left\{\left(x_{0}, x\right) \in \Omega^{\#}: x \in S\right.$ and $\left.x_{0} \leq \xi_{0, *}(\hat{b})-\xi_{0, *}(\hat{a})-\psi(x)\right\}$, where $\psi$ is a smooth function on $\Omega$ that vanishes at $\xi_{*}(\hat{b})$ and is strictly positive everywhere else. Let $\left.\left.C^{\#}=\right]-\infty, 0\right] \times C$. Then $C^{\#}$ is a Boltyanskii approximating cone to $S^{\#}$ at $\xi_{*}^{\#}(\hat{b})$. Furthermore, it is easy to see that the optimality of $\left(\eta_{*}, \xi_{*}^{\#}\right)$ implies that $\mathcal{R}_{\hat{a}, \hat{b}}^{\#}(\hat{x})$ at $\xi_{*}^{\#}(\hat{b})$ and $S^{\#}$ are separated at $\xi_{*}^{\#}(\hat{b})$. Then standard set separation theorems tells us that the cones $\mathcal{C}$ and $C^{\#}$ are not strongly transversal. Since $C^{\#}$ is not a linear subspace, the cones $\mathcal{C}$ and $C^{\#}$ are in fact not transversal. This implies that there exists a nonzero covector $\bar{\omega}^{\#}=\left(\tilde{\omega}_{0}, \bar{\omega} \in T_{\xi_{*}(\hat{b})} \Omega^{\#}\right.$ such that $\left\langle\bar{\omega}^{\#}, z\right\rangle \geq 0$ whenever $z \in C^{\#}$, and $\left\langle\bar{\omega}^{\#}, z\right\rangle \leq 0$ whenever $z \in \mathcal{C}$. It follows that $-\bar{\omega} \in C^{\dagger}$, and also that $\tilde{\omega}_{0} \leq 0 . \omega(t)=\bar{\omega} \cdot D_{\eta_{*}} \Phi_{\hat{b}, t}\left(\xi_{*}(t)\right)+\tilde{\omega}_{0} \nabla c_{\hat{b}, t}\left(\xi_{*}(t)\right)$ for $t \in I$. If $j=1, \ldots, m$, then

$$
\begin{aligned}
0 \geq\left\langle\bar{\omega}^{\#}, w_{j}^{\#}\right\rangle & \left.=\left\langle\left(\tilde{\omega}_{0}, \bar{\omega}\right),\left(v_{0, j}+m_{\hat{b}, t_{j}} \cdot v_{j}, M_{\hat{b}, t_{j}} \cdot v_{j}\right)\right)\right\rangle \\
& =\tilde{\omega}_{0} v_{0, j}+\tilde{\omega}_{0} m_{\hat{b}, t_{j}} \cdot v_{j}+\bar{\omega} \cdot M_{\hat{b}, t_{j}} \cdot v_{j} \\
& =\tilde{\omega}_{0} v_{0, j}+\left(\tilde{\omega}_{0} m_{\hat{b}, t_{j}}+\bar{\omega} \cdot M_{\hat{b}, t_{j}}\right) \cdot v_{j} \\
& =\tilde{\omega}_{0} v_{0, j}+\omega\left(t_{j}\right) \cdot v_{j} .
\end{aligned}
$$

This shows that $\bar{\omega}^{\#} \in \mathcal{K}(\mathcal{W})$, so $\mathcal{K}(\mathcal{W}) \neq \emptyset$, completing our proof.

\section{Variable time problems}

A minimum time problem is, by its very nature, a variable time-interval problem. Hence such a problem does not fit the framework of our main theorem, if we require that the time set $E$ be a subset of $\mathbb{R}$, and that the time from $s$ to $t$ be precisely $t-s$. It is possible, however, to apply Theorem 1 to minimum time problems, and to more general variable time-interval problems, by means of a simple device. Assume that we start with a situation in which $E$ is a subset of $\mathbb{R}$ and our flow-augmentation pairs $\left({ }_{\eta} \Phi,{ }_{\eta} c\right)$ are such that that ${ }_{\eta} c_{t, s}(x)=t-s$ whenever $(s, t) \in E^{\preceq, 2}$. We want to change our point and think of $E$ as representing a "pseudotime" which is no longer physical time, although it will correspond to physical time along the reference trajectory - for example, in the form of a clock that displays at each $t \in E$ the value $t$. For this purpose, 
we allow "insertion variations" in which the reference augmented flow map ${ }_{\eta_{*}} \Phi_{\hat{b}, \hat{a}}^{\#, \eta_{*} c}$ is replaced by the map ${ }_{\eta_{*}} \Phi_{\hat{b}, t}^{\#, \eta_{*} c}{ }_{\eta} \Phi_{t+\varepsilon, t}^{\#, \eta_{\eta_{*}} c} \Phi_{t, \hat{a}}^{\#, \eta_{*} c}$ still regarded mas a transition map from "time" $\hat{a}$ to "time" $\hat{b}$, even though the true physical time $\xi_{0}(\hat{b})-\xi_{0}(\hat{a})$ during which this transition occurs is $\hat{b}-\hat{a}+\varepsilon$. We also allow "deletion variations" in which the reference augmented flow map ${ }_{\eta_{*}} \Phi_{\hat{b}, \hat{a}}^{\#,{ }_{\eta_{*}} c}$ is replaced by the map $\eta_{*} \Phi_{\hat{b}, t}^{\#, \eta_{*} c}{ }_{\eta} \Phi_{t-\varepsilon, \hat{a}}^{\#, \eta_{*} c}$, again regarded as a transition map from "time" $\hat{a}$ to "time" $\hat{b}$, even though the true physical time $\xi_{0}(\hat{b})-\xi_{0}(\hat{a})$ of this transition is $\hat{b}-\hat{a}-\varepsilon$. (Naturally, for this to be possible, we need, for example, to be able to regard $\eta_{*} \Phi_{\hat{b}, t}^{\#, \eta_{*} c}$ as a "time $t+\varepsilon$ to time $\hat{b}+\varepsilon$ " map. The key condition needed for all this to work is to have a time-translation invariant system, that is, a system for which ${ }_{\eta} \Phi_{t, s}={ }_{\eta} \Phi_{t+\alpha, s+\alpha}$ for all $\alpha \in \mathbb{R}$.)

The variational impulses $\left(v^{\#}, t\right)$ that occur in our main theorem are, in general, of a special form. First of all, for each $t \in E$ that occurs in one of the members $\left(v^{\#}, t\right) \in \mathcal{V}$, there exists a vector $v_{\text {del }}^{\#}(t)$, depending on $t$ but not on $v^{\#}$, that corresponds to the "deletion of the reference control on intervals of legth $\varepsilon$." Second, for each $v^{\#} \in \mathcal{V}[t]$-where $\mathcal{V}[t]=\left\{v^{\#}:\left(v^{\#}, t\right) \in \mathcal{V}\right\}$ - the vector $v^{\#}$ corresponds to the "deletion of the reference control on intervals of legth $\varepsilon$ followed by an insertion of some other control on an interval of $\operatorname{lentgh} \varepsilon$, " so that $v_{i n s}^{\#} \stackrel{\text { def }}{=} v^{\#}-v_{\text {del }}^{\#}(t)$ corresponds to an insertion without deletion, and then $v^{\#}=v_{i n s}^{\#}+v_{d e l}^{\#}(t)$. If we allow the insertions to be carried out without a corresponding deletion we get, in addition to the inequalities $\left\langle\omega^{\#}(t), v^{\#}\right\rangle \leq 0$ that occur in (4) of the statement of Theorem 1, the new inequalities $\left\langle\omega^{\#}(t), v_{i n s}^{\#}\right\rangle \leq 0$. If we also allow deletions to be carried out without a corresponding insertion, we get the inequalities $\left\langle\omega^{\#}(t), v_{d e l}^{\#}(t)\right\rangle \leq 0$. On the other hand, one of the controls that can be used in an insertion is the reference control itself, and this insertion corresponds to the vector $-v_{d e l}^{\#}(t)$, yielding the inequality $\left\langle\omega^{\#}(t),-v_{\text {del }}^{\#}(t)\right\rangle \leq 0$. So $\left\langle\omega^{\#}(t), v_{\text {del }}^{\#}(t)\right\rangle=0$. and $\left\langle\omega^{\#}(t),-v_{d e l}^{\#}(t)\right\rangle=0$. In other words,

(\%) for a variable time interval problem where the impulses $\left(v^{\#}, t\right)$ admit the decomposition $v^{\#}=v_{i n s}^{\#}+v_{d e l}^{\#}(t)$ as above, the conclusion of Theorem 1, that $\left\langle\omega^{\#}(t), v^{\#}\right\rangle \leq 0$-which is equivalent to the inequality $\left\langle\omega^{\#}(t), v_{i n s}^{\#}\right\rangle \leq\left\langle\omega^{\#}(t),-v_{d e l}^{\#}(t)\right\rangle-$ can be strengthened to

$$
\left\langle\omega^{\#}(t), v_{i n s}^{\#}\right\rangle \leq\left\langle\omega^{\#}(t),-v_{d e l}^{\#}(t)\right\rangle=0 .
$$

\section{The reflected brachistochrone}

As a our firt example of a nontrivial application of Theorem 1, we briefly outline the results on the "reflected brachistochrone problem" (RBP), studied in detail in [12]. 
The RBP is the minimum time problem for the dynamical law $\dot{x}=u \sqrt{|y|}$, $\dot{y}=v \sqrt{|y|}$, with state $(x, y) \in \mathbb{R}^{2}$ and control $(u, v) \in \mathbb{S}^{1}$ where $\mathbb{S}^{1}$ is the unit circle $\left\{(u, v) \in \mathbb{R}^{2}: u^{2}+v^{2} \leq 1\right\}$. The goal is to characterize the minimum-time trajectory from $A$ to $B$, for any two given points $A=\left(x_{A}, x_{B}\right)$, $B=\left(x_{B}, y_{B}\right)$ in $\mathbb{R}^{2}$.

The result is as follows. If $y_{A} y_{B} \geq 0$, then the optimal trajectory from is obtained by solving the classical (1696-7) brachistochrone problem (BP) of Johann Bernoulli. The solution is an arc of cycloid if $x_{A} \neq x_{B}$, and a straightline segment when $x_{A}=x_{B}$. The most interesting case is when $y_{A} y_{B}<0$. The classical results on the BP tell us that the solution consists of an arc of cycloid $\xi_{1}$ from a to a point $C$ in the $x$ axis, followed by another arc of cycloid $\xi_{2}$ from $C$ to $B$. The point $C$ is determined by applying Theorem 1, and has to be such that the rolling circles that generate the $\xi_{1}$ and $\xi_{2}$ have equal radii.

\section{Snell's law of refraction}

We consider the minimum time problem for the two-dimensional system $\dot{x}=c(x, y) u, \dot{y}=c(x, y) v$, where the control $(u, v)$ takes values in the unit circle $\mathbb{S}^{1}$, and the function $c$ (the "speed of light") is given by $c(x, y)=c_{+}$if $y \geq 0$ and $c(x, y)=c_{-}$if $y<0$. Here $c_{+}$and $c_{-}$are two fixed positive constants such that $c_{+}>c_{-}$. We will focus on the problem of finding a time-minimizing arc from a point $A=\left(x_{A}, y_{A}\right)$ such that $y_{A}>0$ to a point $B=\left(x_{B}, y_{B}\right)$ such that $y_{B}<0$.

The solution of this problem - Snell's law of refraction - is well known, and can be derived by very elementary means: first, one shows that the solution must consist of a straight segment from $A$ to a point $C$ lying on the $x$ axis, followed by the segment from $C$ to $B$; finding $C$ then becomes a rather simple first-year calculus exercise. Here we will show how our version of the Maximum Principle applies to this problem, and leads to Snell's law.

We take our control set $U$ to be the product $\mathbb{S}^{1} \times \mathbb{S}^{1}$, and then define, for each $z=\left(u_{+}, v_{+}, u_{-} . v_{-}\right) \in U$, a discontinuous vector field $X_{z}$ by letting $X_{z}(x, y)=\left(c_{+} u_{+}, c_{+} v_{+}\right)$if $y>0$, and $X_{z}(x, y)=\left(c_{-} u_{-}, c_{-} v_{-}\right)$if $y \leq 0$. We let $G$ be the subset of $U$ consisting of those $\left(u_{+}, v_{+}, u_{-} . v_{-}\right) \in U$ such that $v_{+}<0$ and $v_{-}<0$. We use $L$ to denote the $x$ axis.

An elementary argument shows that an optimal trajectory $\xi_{*}$ must consist of a segment from $A$ to $C$ followed by a segment from $C$ to $B$, where $C \in L$. That is, we can confine ourselves to a trajectory $\xi_{*}:[0, T] \mapsto \mathbb{R}^{2}$ such that (i) $\xi_{*}(0)=A$, (ii) $\left.\xi_{*} T\right)=B$, (iii) $\xi_{*}(\tau) \in L$ for some $\tau$ such that $0<\tau<T$, (iv) if $\xi_{*}(t)=\left(x_{*}(t), y_{*}(t)\right)$ for $t \in[0, T]$, then $y_{*}(t)>0$ for $0 \leq t<\tau$ and $y_{*}(t)<0$ for $\tau<t \leq T$, and (v) the curve $\xi_{*}$ is a trajectory of a constant control $z_{*} \in U$.

All that is left now is to find a condition that will determine $C$. With our choice of $U$, constant controls have two degrees of freedom, but one is removed 
when we stipulate that $\xi_{*}$, starting at $A$, has to go through $B$, so we need to find an extra constraint on $z_{*}$.

Let us compute the flow of $X_{z}$ for a $z \in G$. It suffices to compute the maps $\Phi_{t, 0}^{X,}$, since $\Phi_{t, s}^{X_{z}}=\Phi_{t-s, 0}^{X_{z}}$.

If $y>0, t>0$, and we let $(\tilde{x}, \tilde{y})=\Phi_{t, 0}^{X z}(x, y)$, then $y>\tilde{y}$ and, in addition, $\tilde{y}>0$ as long as $t<\tau_{z}(x, y)$, where $\tau_{z}(x, y)$ is the time for which $\Phi_{\tau_{z}(x, y), 0}^{X z_{z}}(x, y) \in L$. It is clear that $\tau_{z}(x, y)=-\frac{y}{c_{+} v_{+}}$, and also that

$$
\begin{aligned}
\Phi_{t, 0}^{X z}(x, y) & =\left(x+t c_{+} u_{+}, y+t c_{+} v_{+}\right) \text {if } 0<t<\tau_{z}(x, y), \\
\Phi_{\tau_{z}(x, y)+t, 0}^{X_{z}}(x, y) & =\left(x+\tau_{z}(x, y) c_{+} u_{+}+t c_{-} u_{-}, t c_{-} v_{-}\right) \text {if } t>0 .
\end{aligned}
$$

In particular, given a $t$ such that $t \neq \tau_{z}\left(x_{A}, y_{A}\right)$, the flow map $\Phi_{t, 0}^{X_{z}}$ is of class $C^{1}$ near $A$, and is given, for $(x, y)$ in some neighborhood $N(t)$ of $A$, by

$$
\begin{aligned}
\Phi_{t, 0}^{X z}(x, y) & =\left(x+t c_{+} u_{+}, y+t c_{+} v_{+}\right) \text {if } 0<t<\tau_{z}\left(x_{A}, y_{A}\right) \\
\Phi_{t, 0}^{X z}(x, y) & =\left(x+\tau_{z}(x, y) c_{+} u_{+}+\left(t-\tau_{z}(x, y)\right) c_{-} u_{-},\left(t-\tau_{z}(x, y)\right) c_{-} v_{-}\right) \\
& =\left(x-\frac{y}{v_{+}} u_{+}+\left(t+\frac{y}{c_{+} v_{+}}\right) c_{-} u_{-},\left(t+\frac{y}{c_{+} v_{+}}\right) c_{-} v_{-}\right) \\
& =\left(x+\frac{c_{-} u_{-}-c_{+} u_{+}}{c_{+} v_{+}} y+t c_{-} u_{-}, \frac{c_{-} v_{-}}{c_{+} v_{+}} y+t c_{-} v_{-}\right) \text {if } t>\tau_{z}\left(x_{A}, y_{A}\right) .
\end{aligned}
$$

It follows from this that the differential $D^{z}(t)$ of $\Phi_{t, 0}^{X_{z}}$ at $A$ is given by $D^{z}(t)=\mathbb{I}_{\mathbb{R}^{2}}$ if $t<-\frac{y_{A}}{c_{+} v_{+}}$and $D^{z}(t)=M_{z}$ if $t>-\frac{y_{A}}{c_{+} v_{+}}$, where $M_{z}$ is the matrix $\left[\begin{array}{ll}1 & \frac{c_{-} u_{-}-c_{+} u_{+}}{c_{=} v_{+}} \\ 0 & \frac{c_{-}}{c_{+} v_{+}}\end{array}\right]$.

We now let $\hat{D}(t)$ denote the differential of the flow map $\Phi_{T, t}^{X_{z_{*}}}$ at $\xi_{*}(t)$, where $\xi_{*}:[0, T] \mapsto \in \mathbb{R}^{2}$ is our reference trajectory $z_{*}=\left(u_{*,+}, v_{*,+}, u_{*,-} . v_{*,-}\right)$ is our constant reference control, and It is then clear that $\hat{D}(t)=D^{z_{*}}(T) D^{z_{*}}(t)^{-1}$, and then $\hat{D}(t)=\mathbb{I}_{\mathbb{R}^{2}}$ if $t>-\frac{y_{A}}{c_{+} v_{*+}}$, and $\hat{D}(t)=M_{z_{*}}$ if $t<-\frac{y_{A}}{c_{+} v_{*+}}$.

To apply our flow version of the Maximum Principle, we take the time set $E$ to be $[0, T] \backslash\left\{\tau_{z_{*}}(A)\right\}$. Then each flow map $\Phi_{t, s}^{X_{z_{*}}}$ is of class $C^{1}$ (and, in fact, real analytic) on a neighborhood of $\xi_{*}(s)$, as long as $s, t \in E$ and $s \leq t$.

Let $\omega$ be the adjoint vector given by the Maximum Principle. Then $\omega(t)=\bar{\omega}^{-}$if $t>-\frac{y_{A}}{c_{+} v_{*,+}}$, and $\omega(t)=\bar{\omega}^{+}$if $t<-\frac{y_{A}}{c_{+} v_{*,+}}$, where $\bar{\omega}^{-}=\left(\bar{\omega}_{x}, \bar{\omega}_{y}\right)$, $\bar{\omega}^{+}=\left(\bar{\omega}_{x}, \hat{\omega}_{y}\right)$, and $\hat{\omega}_{y}=\frac{1}{c_{+} v_{*,+}}\left(\left(c_{-} u_{*,-}-c_{+} u_{*,+}\right) \bar{\omega}_{x}+c_{-} v_{*,-} \bar{\omega}_{y}\right)$. The Hamiltonian maximization condition of the Maximum Principle implies that $\omega^{-}$must be a scalar multiple of $\left(u_{*,-}, v_{*,-}\right)$, and $\omega^{+}$has to be a scalar multiple of $\left(u_{*,+}, v_{*,+}\right)$. This means that $\bar{\omega}_{x}=k_{-} u_{*,-}=k_{+} u_{*,+}, \bar{\omega}_{y}=k_{-} v_{*,-}$, and $\hat{\omega}_{y}=k_{+} v_{*,+}$ for some positive constants $k_{-}, k_{+}$.

It follows that $\frac{k_{-}}{k_{+}}=\frac{u_{*,+}}{u_{*,-}}$. Let $\omega_{0}$ be the abnormal multiplier. Then

$$
0=\left\langle\bar{\omega}^{-}, c_{-}\left(u_{*,-}, v_{*,-}\right)\right\rangle-\omega_{0}=\left\langle\bar{\omega}^{+}, c_{+}\left(u_{*,+}, v_{*,+}\right)\right\rangle-\omega_{0},
$$


so $\left\langle\bar{\omega}^{-}, c_{-}\left(u_{*,-}, v_{*,-}\right)\right\rangle=\left\langle\bar{\omega}^{+}, c_{+}\left(u_{*,+}, v_{*,+}\right)\right\rangle$.

Furthermore, $\bar{\omega}^{-}=k_{-}\left(u_{*,-}, v_{*,-}\right), \quad \bar{\omega}^{+}=k_{+}\left(u_{*,+}, v_{*,+}\right)$, and both $\left(u_{*,-}, v_{*,-}\right)$, and $\left(u_{*,+}, v_{*,+}\right)$ are unit vectors. It follows that $k_{-} c_{-}=k_{+} c_{+}$. Hence $\frac{k_{-}}{k_{+}}=\frac{c_{+}}{c_{-}}$. Therefore $\frac{u_{*,+}}{u_{*,-}}=\frac{c_{+}}{c_{-}}$.

Let $\theta_{i}$ be the "angle of incidence," that is, the angle between the line $A C$ and the $y$ axis. Let $\theta_{r}$ be the "angle of refraction," that is, the angle between the line $C B$ and the $y$ axis. It is then clear that $u_{*,+}=\sin \theta_{i}$ and $u_{*,-}=\sin \theta_{r}$. Then

$$
\frac{\sin \theta_{i}}{\sin \theta_{r}}=\frac{c_{+}}{c_{-}},
$$

which is precisely Snell's law.

\section{References}

1. Pontryagin L S, Boltyanskii V G, Gamkrelidze R V, Mischenko E F (1962) The Mathematical Theory of Optimal Processes. Wiley, New York.

2. Clarke F H (1976) The Maximum Principle under minimal hypotheses. SIAM J. Control Optim. 14:1078-1091.

3. Clarke F H, Ledyaev Yu S, Stern R J, Wolenski P R (1998) Nonsmooth Analysis and Control Theory. Springer Verlag, Graduate Texts in Mathematics No. 178; New York.

4. Clarke F H (2005) Necessary conditions in dynamic optimization. Memoirs Amer. Math. Soc. 816, vol. 173.

5. Ioffe A (1997) Euler-Lagrange and Hamiltonian formalisms in dynamic optimization. Trans. Amer. Math. Soc. 349:2871-2900

6. Ioffe A, Rockafellar R T (1996) The Euler and Weierstrass conditions for nonsmooth variational problems. Calc. Var. Partial Differential Equations 4:59-87.

7. Knobloch H W (1975) High Order Necessary Conditions in Optimal Control. Springer, Berlin.

8. Krener A J (1977) The High Order Maximal Principle and Its Application to Singular Extremals. SIAM J. Contrl. Optim. 15:256-293.

9. Mordukhovich B (2006) Variational Analysis and Generalized Differentiation, I: Basic Theory; II: Applications. Grundlehren Series (Fundamental Principles of Mathematical Sciences), Vols. 330 and 331. Springer, Berlin.

10. Sussmann H J (1994) A strong version of the Łojasiewicz Maximum Principle. In: Pavel N H (ed) Optimal Control of Differential Equations, (Athens, OH, 1993). Lect. Notes in Pure and Applied Math. 160:293-309; Marcel Dekker, New York.

11. Sussmann H J (2000) New theories of set-valued differentials and new versions of the maximum principle of optimal control theory. In: Isidori A, LamnabhiLagarrigue F, Respondek W (eds), Nonlinear Control in the year 2000, 487-526. Springer, London, 2000.

12. Sussmann H J (2004) Optimal control of nonsmooth systems with classically differentiable flow maps. In: Proc. 6th IFAC Symposium on Nonlinear Control Systems (NOLCOS 2004), Stuttgart, Germany, September 1-3, 2004. Vol. 2:pp. 609-704.

13. Vinter R B (2000) Optimal Control. Birkhäuser, Boston. 